\title{
Modifications récentes de la végétation aquatique de l'étang du Vaccarès (Camargue, France) liées aux perturbations anthropiques
}

\author{
A. Vaquer 1 \\ P. Heurteaux!
}

Mots clés: Camargue, Vaccarès, écosystème perturbé, végétation aquatique, transmission de la lumière.

L'étang du Vaccarès est isolé des influences naturelles de la mer et du Rhône depuis la construction de digues au siècle dernier. Depuis 1950, des modifications de la salinité liées aux conditions climatiques et aux activités humaines ont ent raîné des changements remarquables de sa végétation aquatique. Les transformations des herbiers ont pu être cartographiées grâce à des relevés effectués en 1963-64, 1974 et 1984. L'évolution saisonnière da la salinité, la bathymétrie, la nature du substrat et les qualités optiques de l'eau permettent d'interpréter les modifications de la végétation.

Pendant la phase de dessalement, la végétation à Potamogeton pectinatus et Ruppia maritima évolue vers une végétation dulçaquicole à Myriophyllym spicatum et Phragmites communis. A partir de 1980, l'augmentation de la salinité a permis l'installation d'un herbier monospecifique à Zostera noltii.

Par leur action sur la dispersion et la floculation des sédiments, les variations de salinité, ajoutées à l'action des vents fréquents en Camargue, influent sur la transparence de l'eau et la transmission de la lumière dans l'eau. Une meilleure transmission de la lumière serait une explication de la plus grande extension des herbiers pendant la phase de resalement du Vaccarès.

L'avenir de cette végétation reste entièrement liée à la salinité dont les variations dépendent des conditions climatiques et des activités humaines.

Recent modifications of the aquatic vegetation in the Ktang du Vaccarès (Camargue, France) in relution with the human disturbances.

Keywords : Camargue, Vaccarès, disturbed ecosystem, aquatic vegetation, light transmission.

Since the construction of the dykes in the last century, the Vaccarès, which is the main waterbody on the Reserve Nationale de Camargue, has been cutoff from the natural influences of the sea and the Rhone. Since then, apar from the uncontrolled action of climatic conditions (rainfall and evaporation) water and salt balance have been basically cont rolled activities, whereas salinity has been and is still subjected to fundamental changes. Prior to 1950, it was very saline (up to $100 \mathrm{~g} . \mathrm{l}^{-1}$ ); it became salty between 1950 and 1979 (mean salinity $6 \mathrm{~g} .1-1$ ), then from 1980 its salinity increased again to reach values close to sea water as from 1984. Since 1950 these changes in salinity have divertly led to striking changes in the aquatic plant community, which was monitored and mapped in 1963-1964, 1974 and 1984. Salinity changes over this period, bathymetry substratum composition and optical quality of water can be used to interpret the evolution of the vegetation.

During the desalination period the halophytic vegetation, including Potamogeton pectinatus and Ruppia maritima, tended to a freshwater type with Myriophyllum spicatum and Phragmites communis expanding whereas Ruppia maritima disappeared completely. As soon as 1980 as a consequence of the increase in salinity all these species disappeared and two years later they had been replaced by Zostera noltit. Salinity changes indirectly acted on water quality especially in its clarity. During the desalination period large amounts of muddy deposit on the substratum were defloculated and maintained in suspension by the turbulent action of very frequent winds $\left(91\right.$ and $247 \mathrm{mg} .1^{-1} \mathrm{TSM}^{-} \mathrm{yr}^{-1}$ on average in 1965 and 1978). In 1978, the calculated compensation limit would have reached a depth of $0.6 \mathrm{~m}$. Then the quantity of light transmit ted was probably a limiting factor to the development of aquatic plant communities. Increasing salinity favoured Flocculation of TSM (in 1984, the day after windy days, $12.8 \mathrm{mg} . \mathrm{l}^{-1}$ on average). The bet ter transmission of the light through the water (with a compensation limit exceeding the depth of the waterbody) would explain a larger ex tension of the aquatic plant community during the last period.

The future of this vegetation is closely connected with the variations in salinity depending in turn on climatic conditions and human activity.

\footnotetext{
1. C.N.R.S., Laboratoire d'Hydrobiologie, Université de Provence, 3 place V. Hugo, 13331 Marseille Cédex 3.
} 


\section{Introduction}

L'étang du Vaccarès est le principal élément d'une vaste mosaïque de milieux aquatiques et terrestres halomorphes constituant depuis 1927 la Réserve Nationale de Camargue. L'intérêt biologique et écologique de cet te région a largement dépassé les frontières régionales et nationales. Cet étang se trouve maintenant au cceur d'une réserve mondiale de Biosphère. En dépit d'un statut de protection normalement favorable à sa conservation, l'étang du Vaccarès a subi et subit encore actuellement l'influence d'intérêts le plus souvent divergents, héritage d'un passe historique, social et économique de plusieurs siècles.

Cette influence pèse sur les bilans hydrique et salin, et a pour conséquence des modifications radicales de la salinité du milieu. Ainsi avant 1950, il s'agissait d'un étang salé à sursalé avec une très forte euryhalinité périodique (salinité pouvant atteindre $100 \mathrm{~g} \cdot \mathrm{l}^{-1}$ au cours d'étés très secs et 10 g. $1^{-1}$ au cours d'hivers pluvieux), les niveaux d'eau moyens étaient assez bas, avec un marnage saisonnier pouvant étre important $(>1 \mathrm{~m})$ et se traduire par des périodes d'assec. Au cours des quarante dernières années, le Vaccarès a connu, pour des raisons qui seront analysées plus loin, deux de ces modifications :

1) de 1950 à 1979, la salinité a baissé et l'étang est devenu faiblement saumâtre à la fin de la décennie 1970 (6 g.l-1 de salinité moyenne annuelle) : l'euryhalinité s'est estompée, les niveaux moyens sont restés élevés et le marnage saisonnier peu marqué (de 0,40 à $0,90 \mathrm{~m}$ ) :

2) à partir de 1980, la salinité a fortement augmenté et atteint des valeurs submarines dès 1984 , avec des écarts saisonniers relativement faibles; les niveaux moyens sont restés hauts et le marnage saisonnier tamponné.

De telles modifications du milieu physique président à de grands bouleversements écologiques. Les biocénoses subissent de profondes transformations, comme c'est le cas par exemple pour la faune ichtyologique (Petit \& Schachter 1954) où apparaissent à partir de 1950 des poissons typiquement d'eau douce comme le brochet ou le sandre.

L'objet de cette étude est précisément une mise au point sur les transformations de la végétation aquatique en relation directe ou indirecte avec l'évolution de la salinité.

\section{L'étang du Vaccarès : site et dynamique de l'eau et du sel}

L'étang du Vaccarès occupe au centre de l'île de Camargue une dépression de 6700 hectares, très peu profonde et dont le niveau est situé au-dessous du niveau de la mer. A la côte zéro (NGF), sa profondeur moyenne est de $1.4 \mathrm{~m}$ et sa profondeur maximale de 2 mètres, son volume est de $93.10^{6} \mathrm{~m}^{3}$. Cette dépression résulte du colmatage flandrien d'une lagune plus ou moins ouverte sur la mer. Elle est encadrée à l'est, au nord et à l'ouest par des formations fluviatiles limoneuses ou limono-sableuses et au sud par des formations dunaires sableuses. Les formations fluviales sont les vestiges de deux anciens cours du Rhône atterris au $16^{e}$ sieccle : le Rhône d'Ulmet à l'est et le Rhône de St Férréol au nord et à l'ouest. Les formations dunaires sont les vestiges d'un ancien cordon lit toral arrasé et en partie recouvert par des sédiments lagunaires récents, matérialisé par la presqu'île de Mornès. Au fil des temps, ces formations ceinturant le Vaccarès ont été entaillées par l'action des eaux de l'étang, renforcée par les vents dominants de nord-ouest (mistral) et de sud-est (marin). Cette érosion fort active, soulignée en maints endroits par de petites falaises d'une hauteur de l'ordre du mètre, a nécessité la mise en place d'ouvrages de défense.

Le Vaccarès collecte les effluents d'un réseau de canaux qui draine un bassin versant terrestre de 31500 ha dont environ 15000 sont cultivés.

D'origine lagunaire, le Vaccarès est un étang naturellement salé en contact avec la mer par l'in termédiaire des étangs inférieurs. Son bilan hydrique et salin dépend en premier lieu des conditions climatiques, mais à l'action antagoniste des pluies et de l'évaporation s'ajoutent: 1) les entrées d'eaux de drainage agricole douceâtres et éventuellement les entrées volontaires ou accidentelles d'eau de mer, 2) les sorties du trop-plein des étangs à la mer. Le bilan hydrique et salin du Vaccarès penche tantôt en faveur des entrées, tantôt en faveur des sorties d'eau et de sels. De ce fait, le Vaccarès est un étang à salinité et à niveau variables dans le temps, à l'échelle saisonnière, annuelle et pluriannuelle (fig. 1) 


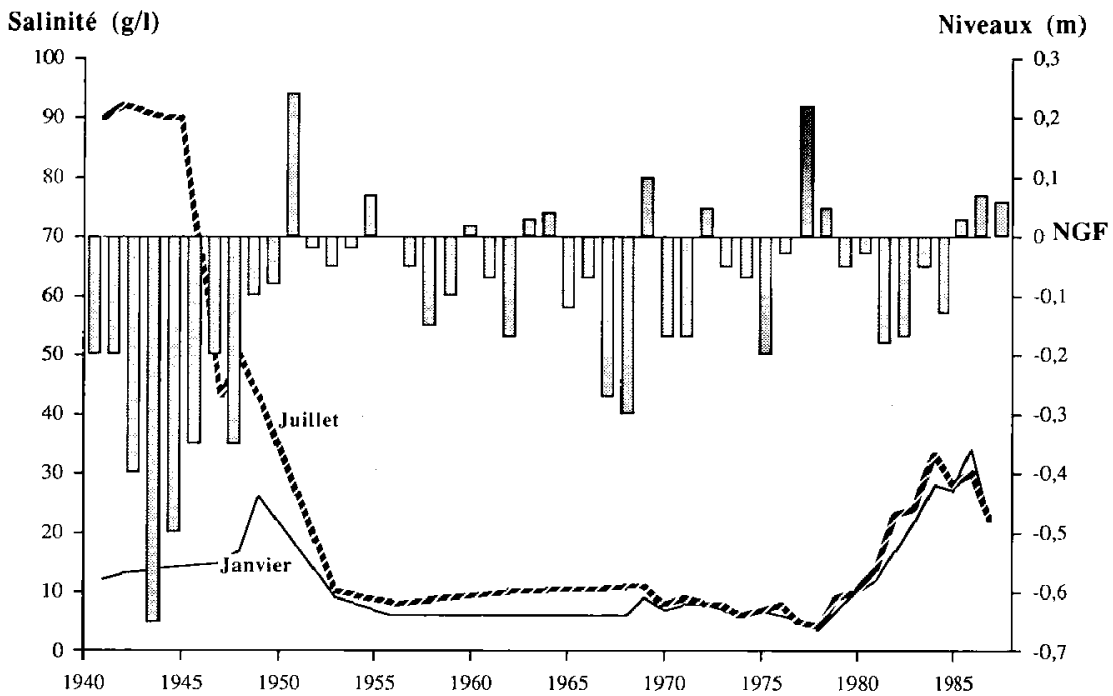

Fig. 1. Evolution de la salinité aux mois de janvier et juillet, et niveaux moyens de l'étang du Vaccarès par rapport au zéro NGF de 1941 à 1987.

Avant la construction des digues protégeant l'île de Camargue des intrusions marines (1859) et rhodaniennes (1869), des entrées imprévisibles et souvent massives d'eau de mer (tempêtes) ou d'eau du Rhòne (inondations) oblitéraient l'antagonisme précipitations-évaporation et faisaient du Vaccarès un biotope très instable et euryhalin, les variations de niveau et de salinité n'avaient pas de caractère périodique.

Après l'endiguement, les entrées et les sorties d'eau de mer et les entrées d'eau du Rhône étant contrôlées, l'antagonisme précipitation-évaporation devint prépondérant. Le régime hydrique el salin du Vaccarès varia au rythme des années sèches et humides et des contrastes saisonniers. Mais le déficit hydrique climatique étant une tendance moyenne pour la Camargue, les niveaux moyens baissèrent et la salinité augmenta. Le Vaccarès devint alors un biotope salé à sursalé, très euryhalin à l'échelle saisonnière et pluriannuelle. Ces caractéristiques, bien que tempérées dès la fin du $19^{e}$ siècle par des apports non négligeables d'eau d'origine viticole (lutte contre le Phylloxera), persistèrent jusqu'à la fin de la décennie 1940.

L'après-guerre fut marquée par l'essor de la riziculture camarguaise et les grands bouleversements hydrauliques qu'il entraîna. L'apport massif des retours d'irrigation rizicoles au Vaccarès provoqua un fort dessalement et une élévation du niveau des étangs favorable à l'évacuation à la mer de leur trop plein, donc aux hémorragies de sel. Le Vaccarès devint alors un biotope faiblement saumâtre aux variations saisonnières de niveau et de salinité très atténuées. Au cours de la décennie 1970, le déclin de la riziculture est compensé par des excédents pluviaux.

A partir de 1979, des entrées d'eau de mer, volontaires ou non, provoquèrent une forte augmentation du stock de sels dissous, donc de la salinité, et transformèrent le Vaccarès en milieu submarin aux variations saisomières de salinité peu marquées. Depuis 


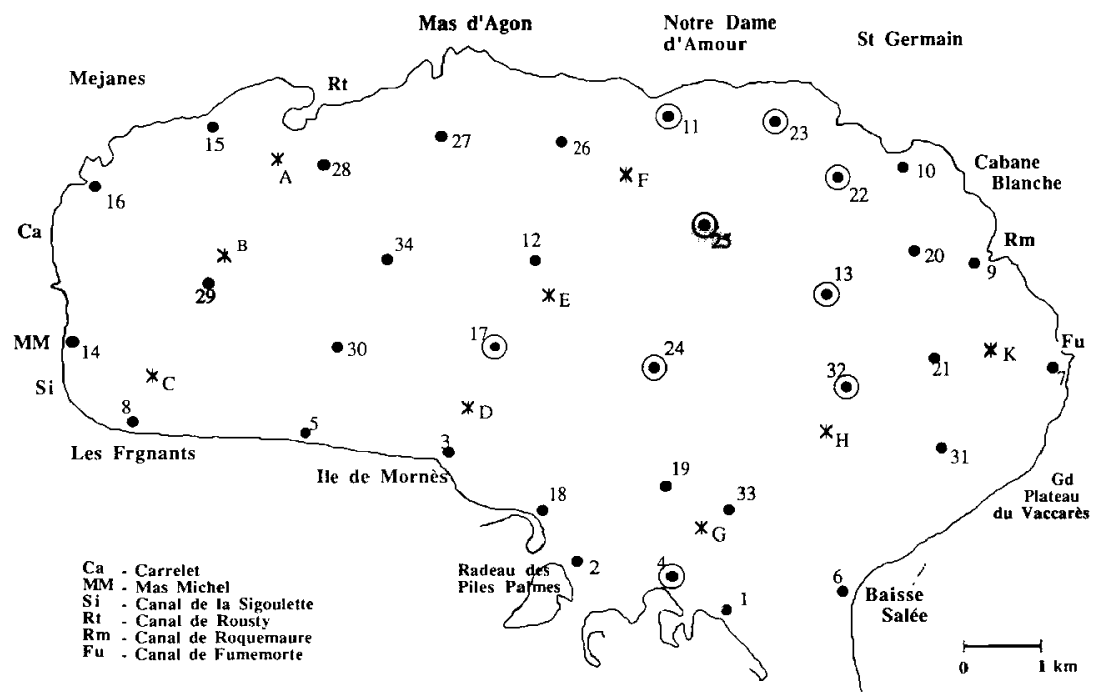

Fig. 2. Localisation des stations expérimentales sur l'étang du Vaccarès en 1984. Points noirs : les 34 stations de prélèvements de sédiments du fond (aux 9 stations cerclées, la couche de sédiments non consolidés est absente ou très mince). Etoile : les 9 stations d'étude de la transmission de la lumière.

1986, trois hivers successifs pluvieux et la reprise de la riziculture, favorables aux hauts niveaux et aux sorties de sels à la mer ont tempéré cette tendance.

\section{Méthodes}

Les mesures de la salinité ont été faites sur le Vaccarès par plusieurs personnes ou organismes utilisant des procédés et modes d'expression différents (densimétrie, dosages des halogénures par la méthode de Mohr ou par coulométrie, conductimétrie, réfracto métrie, résidus secs, balances ioniques). Pour rendre les différentes mesures comparables, elles ont toutes été converties en salinité totale exprimée en grammes de soluté par litre de solution (S g.1-1). Cette conversion a été rendue possible du fait des excellentes corrélations existant entre la salinité totale et les résultats des différents procédés d'analyse.

Les sédiments non consolidés du fond de l'étang, constituant le substrat des herbiers, ont été étudiés en 1984, en 34 points uniformément répartis sur toute l'étendue du plan d'eau (fig. 2). Ils sont quasiment absents en 9 de ces 34 points. Chaque fois qu'ils sont présents, ils sont prélevés par carottage sur toute leur épaisseur, puis homogénéisés et séchés à $105^{\circ} \mathrm{C}$. Leur texture est analysée par tamisage sous l'eau pour séparer la fraction fine $(<50 \mu \mathrm{m})$ de la fraction grossière. $\mathrm{La}$ granulométrie de cette dernière est réalisée par tamisage à sec sur colonne de tamis de 500,250 et $100 \mu \mathrm{m}$.

Les mesures de lumière ont été effectuées en 1984 également, tous les $10 \mathrm{~cm}$ le long de profils verticaux 
à l'aide d'un radiomètre (LI COR 85A) couplé à une sonde du type Underwater Quantum Sensor (LI COR 192S). A chaque niveau les flux de photons $(\mu \mathrm{E}$ $\mathrm{m}^{-2} \mathrm{sec}^{-1}$ ) sont exprimés en pourcentage de la valeur Io mesurée juste sous l'interface eau-air. Trois groupes de trois stations ont été choisis respectivement dans la zone Ouest, dans la partie cen. trale et dans la zone Est de l'étang (fig. 2).

Les quantités de matières en suspension totales (MEST) dans l'eau ont été déterminées par pesées sur des échantillons prélevés dans les 9 stations précédemment choisies, passés sur des filtres Whatman $\mathrm{GF} / \mathrm{F}\left(0,7 \mu \mathrm{m}\right.$ de porosité) et séchés à $105^{\circ} \mathrm{C}$ pendant 24 beures.

En 1963-64, la végétation a été relevée au cours d'une étude bathymétrique faite par Heurteaux et Marazanof. Les observations ponctuelles positionnées par relèvements au compas (et portées sur la carte) ne permettent pas de cartographier précisément des zones de densités différentes. Elles donnent cependant une bonne information sur la limite d'extension et la distribution des espèces.

En 1974 et 1984, les relevés de végétation ont été faits par observation directe, souvent facilitée, compte tenu de la faible profondeur de l'étang, par l'affleurement des plantes à la surface du plan d'eau, et à l'aide d'échantillons prélevés avec un grappin en suivant la limite d'extension des herbiers. Celleci a été régulièrement déterminée en faisant le point par recoupement de relèvements effectués à l'aide d'un compas de repères connus autour de l'étang. Le recouvrement des herbiers a été évalué suivant 3 classes $>50 \%, 10$ à $50 \%$ et $<10 \%$ ) le long de transects perpendiculaires à la rive et espacés d'environ $500 \mathrm{~m}$. Les mesures de profondeur sont faites en même temps que la collecte des végétaux. En 1974, un survol aérien durant l'été a permis de compléter et de préciser les observations au sol.

\section{Résultats}

\subsection{La nature du substrat de l'étang}

Au plan morphologique, le fond du Vaccarès présente deux zones situées de part et d'autre d'une ligne passant à quelque $500 \mathrm{~m}$ au sud des embouchures des canaux de la Sigoulette à l'ouest et de Fumemorte à l'est et marquée par un talus relativement pentu $(0,05 \%)$. Au nord de cette ligne, le fond de l'étang se présente comme une cuvette à fond pratiquement plat (pente $0,01 \%$ ), au sud s'étend un plateau au microrelief assez tourmenté, car sillonné d'anciens chenaux de communication avec les étangs inférieurs et la mer (fig. 3).

L'assise sédimentaire en place au fond de la cuvette du Vaccarès est recouverte d'une couche de sédiments remaniés, non consolidés. L'épaisseur de cette couche varie dans l'espace de 35 à $300 \mathrm{~mm}$ (103 mm en moyenne; $\mathrm{s}=74 ; \mathrm{n}=25$ ) sauf dans 9 stations au centre de l'étang où elle est très mince ou quasiment inexistante. Le matériau constitutif est plus ou moins fin et plus ou moins vaseux (Tableau I). La fraction la plus fine $(<50 \mu \mathrm{m})$ représente en moyenne $38.4 \%$ de ce matériau. Ces sédiments non consolidés peuvent avoir plusieurs origines. Il peut s'agir d'argiles et de limons fins charriés par les eaux de drainage qui apportent à l'étang des eaux de ruissellement pluviales et agricoles toujours turbides. Il peut s'agir également d'argiles arrachées au fond de la cuvette, de limons et de sables arrachés aux berges par l'érosion, ou de débris végétaux (herbiers) plus ou moins décomposés ou encore de débris de test de mollusques (Cerastoderma edule, Abra ovata) plus ou moins pulvérisés.

Tableau I. Distribution moyenne des sédiments meubles du fond de l'étang du Vaccarès selon 5 classes de tailles (en \%)

\begin{tabular}{lccc}
\hline & $\mathrm{n}$ & Moyenne/Ecart type & Etendue \\
\hline$>500 \mu$ & 25 & $9,7 / 11,3$ & $1,0-42,3$ \\
250 à $500 \mu$ & 25 & $10,1 / 7,6$ & $0,4-28,2$ \\
100 ̀े $250 \mu$ & 25 & $36,0 / 23,3$ & $1,3-76,1$ \\
50 a $100 \mu$ & 25 & $5,8 / 6,4$ & $0,9-29,6$ \\
$<50 \mu$ & 25 & $38,4 / 31,4$ & $4,6-95,6$ \\
\hline
\end{tabular}

La répartition spatiale des sédiments non consolidés (fig. 4) met l'accent sur l'importance de l'apport par les eaux de drainage. Les accumulations les plus épaisses se situent en effet dans les zones d'influence des canaux de drainage. A l'est, les apports des canaux de Fumemorte et de Roquemaure sont déportés vers le sud, sans doute sous l'influence des courants animant la masse d'eau, fortement soumise à l'action des vents. Ces courants n'ont toutefois jamais été matérialisés par des mesures. A l'ouest, les canaux de Rousty et de la Sigoulette ne s'écoulent plus au Vaccarès depuis une dizaine d'années, 

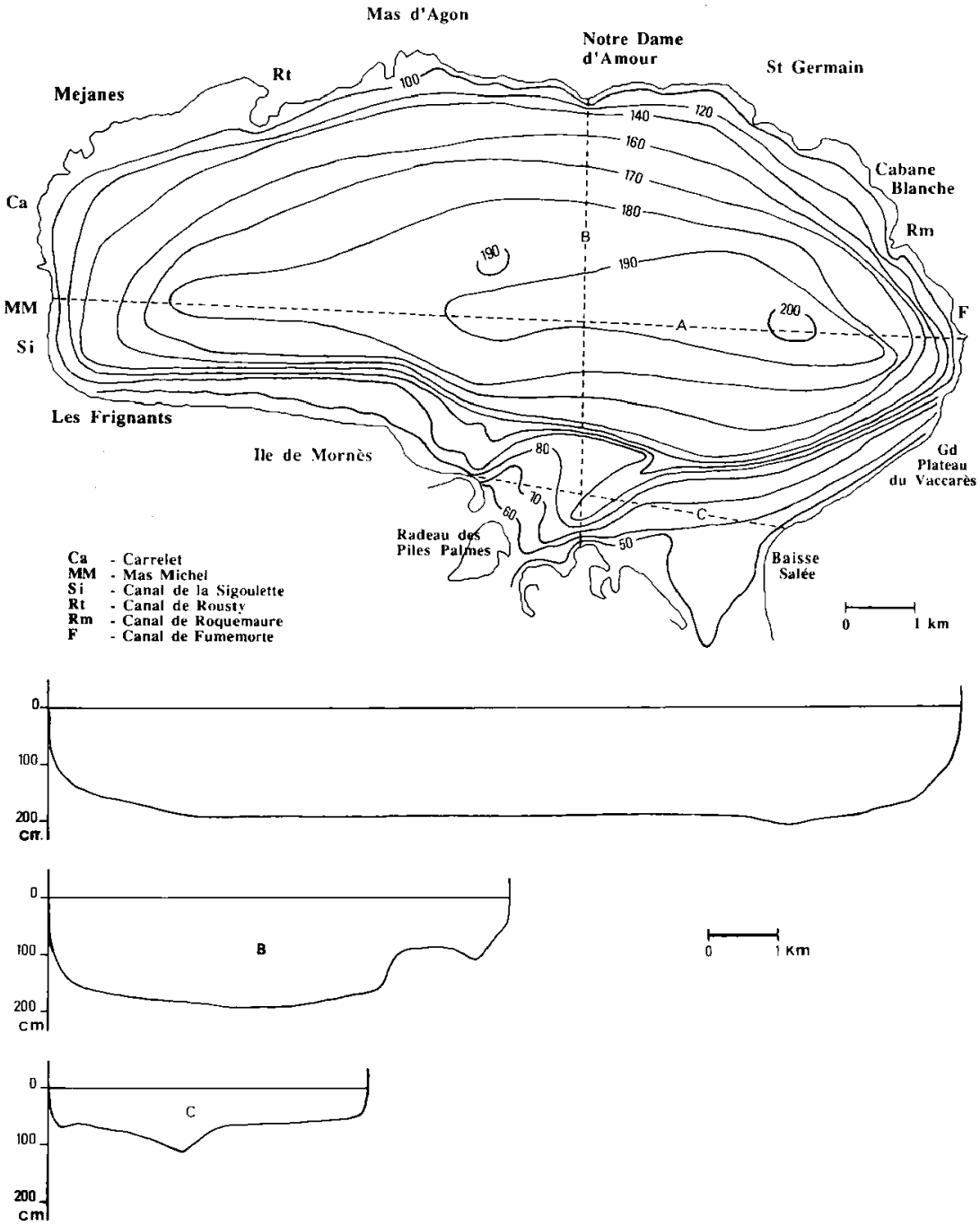

Fig. 3. Bathymétrie de l'étang du Vaccarès d'après la carte au 1/10000 dressée par la Réserve Nationale de Camargue (reproduite avec l'aimable autorisation d'E. Coulet, Directeur de la RNC). A, B, C profils du fond de l'étang. 
..7 Vase limono-argileuse, microcalcaire

$[\because \ldots]$ et organique

$\because:$ Sables coquilliers

Sables fins limoneux provenant de l'érosion des berges
[:-

$\therefore$ Sédiments sablo-limoneux micro-calcaires attribués aux appons par les canaux

Absence de sédiments meubles

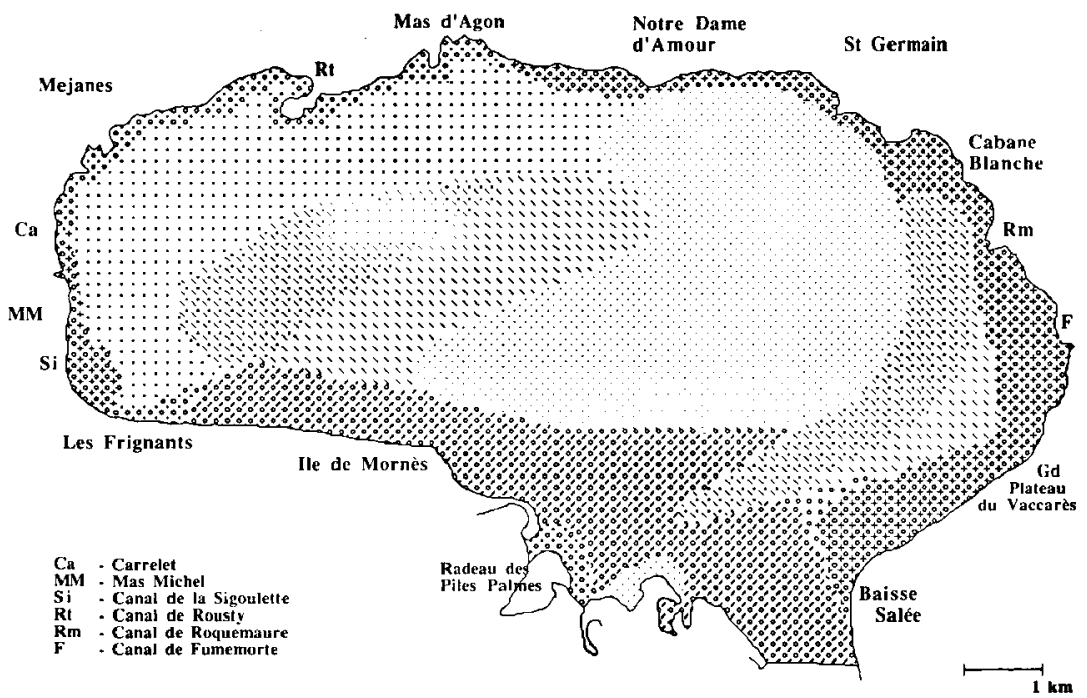

Fig. 4. Répartition des sédiments non consolidés au fond du Vaccarès

leurs apports solides antérieurs semblent avoir subi un fort remaniement rhéologique. Le róle de pièges joué par les herbiers passés et présents se manifeste également par l'accumulation des sédiments dans la zone d'extension des herbiers en bordure de l'ètang et leur quasi absence dans la zone centrale où les macrophytes ne se sont jamais développés.

La granulométrie du matériau dépend beaucoup de son origine. Les argiles arrachés au bassin versant et au fond de l'étang et la matière organique décomposée donnent des vases riches en colloides. comme dans la zone ouest alors que les sables dunaires et les débris de coquilles constituent la matrice minérale de vases plus grossières comme on en rencontre le long de la côte est de celle de Mornès.

\subsection{Les qualités optiques de l'eau}

Par forts vents, la lame d'eau de l'étang, peu épaisse, est le siège de turbulences sur toute sa hauteur. Cette agitation remet fréquemment en suspension la couche superficielle des sédiments non consolidés. A titre d'exemple, sur la période de 1963 à 1973, le temps calme n'a représenté en moyenne que $20 \%$ du temps, tandis que les périodes de vents ( $80 \%$ du temps au total) sont partagées en $51 \%$ pour les vents de secteur Nord et $29 \%$ pour les vents de secteur Sud (Heurteaux \& Servant 1979).

Ainsi, jusqu'à la dernière phase de resalement de l'eau dans les années 80 , l'étang du Vaccarès présentait très souvent un aspect très turbide. A 
Tableau II. Coefficients d'extinction de la lumière déterminés par ajustement à la loi de Lambert-Beer et profondeurs calculées correspondant respectivement à 4 et $1 \%$ de la humière incidente, et quantités moyennes des matières en suspension totales.

\begin{tabular}{|c|c|c|c|c|c|c|}
\hline & Stations & Coefficients & r(corr.) & $Z(4 \%)$ & $Z(1 \%)$ & MEST (mg/l) \\
\hline & B & 3,45 & 0,93 & $-1,02$ & $-1,37$ & 12,51 \\
\hline Série de mesures & D & 3,52 & 0,91 & $-1,04$ & $-1,37$ & 19,69 \\
\hline après une période & $\mathbf{E}$ & 1,34 & 1,00 & $-2,42$ & $-3,45$ & 15,07 \\
\hline \multirow[t]{2}{*}{ de vent de Nord } & $\mathrm{F}$ & 1,10 & 1,00 & $-2,91$ & 4,17 & 19,64 \\
\hline & $\mathbf{K}$ & 1,24 & 1,00 & $-2,57$ & $-3,68$ & 11,48 \\
\hline \multirow[t]{4}{*}{ Moyennes } & & 2,17 & & $-1,82$ & $-2,57$ & 16,32 \\
\hline & $\mathbf{A}$ & 0,75 & 0,99 & $-4,25$ & $-6,06$ & 4,70 \\
\hline & $\mathbf{B}$ & 1,21 & 1,00 & $-2,67$ & $-3,81$ & 6,40 \\
\hline & C & 1,47 & 1,00 & $-2,20$ & $-3,14$ & 8,00 \\
\hline & $\mathbf{K}$ & 1,47 & 1,00 & $.2,24$ & $-3,18$ & 10,95 \\
\hline Moyennes & & $t, 61$ & & $-2,26$ & $-3,21$ & 8,89 \\
\hline Moyennes générales & & 1,90 & & $-2,04$ & $-2,89$ & 12,80 \\
\hline
\end{tabular}

l'inverse, depuis l'augmentation de la salinité de l'eau favorisant la floculation des matières en suspension, les eaux sont devenues très transparentes. Il n'existe malheureusement aucune mesure de transmission de la lumière dans l'eau antérieure à 1984 permettant d'évaluer et de comparer les qualités optiques à ces différentes périodes.

En 1984, les 2 séries de profils de transmission de lumière dans l'eau ont été établies en juin, l'une au lendemain d'une journée de mistral et la seconde après une période de vents de secteur sud (Tableau II). Pendant les mesures, les quantités moyennes de lumière incidente étaient de $1944{ }_{\mu} \mathrm{E} \cdot \mathrm{m}^{-2} \cdot \mathrm{sec}^{-1}$ et de $1450 \mu \mathrm{E} \cdot \mathrm{m}^{-2}$. $\mathrm{sec}^{-1}$ juste sous la surface (Io). La quantité moyenne de lumière transmise (I) au fond était de $10,7 \%$ de Io (entre 0,4 et $42,4 \%$ ) pour une profondeur $(Z)$ moyenne de $1,43 \mathrm{~m}$ (entre $1,2 \mathrm{~m}$ et $1,7 \mathrm{~m}$ selon les stations). La transmission de l'énergie lumineuse dans l'eau de l'étang s'effectue suivant la loi classique de Beer-Lambert : $1 / 10=e-\mathcal{E}_{\mathrm{z}}$. La valeur moyenne élevée des coefficients d'extinction $\mathcal{E}\left(1,9 \mathrm{~m}^{-1}\right.$, fluctuant entre 0,75 et $3,52 \mathrm{~m}^{-1}$ (Tableau II) indique une très faible transparence de l'eau. Celle-ci est normalement liée avec les teneurs en matières en suspension, mais leurs modestes quantités (12,8 mg.l-1 en moyenne, comprises entre 4,7 et 28,1 mg.l-1) n'expliquent que pour une faible partie la mauvaise transmission de la lumière comme l'at teste le coefficient de corrélation certes significatif mais peu élevé entre $\mathcal{E}$ et MES $(r=0,49$; $\mathrm{n}=18$ ). Suivant Kirk (1980) et Wamsley et al (1980), la qualité des MES doít jouer dans ces cas un rôle plus fondamental que leur quantité. L'apparente clarté de l'eatu est sans doute liée à la sédimentation assez rapide des grosses particules et des floculats, tandis que la mauvaise transmission serait liée aux particules en suspension les plus fines qui sédimentent très lentement.

Les excellents ajustements de la transmission de la lumière à la loi Lambert-Beer permettent de calculer les profondeurs théoriques où parviennent les quantités de lumière minimales, comprises entre 4 et $1 \%$ de la lumière incidente, pour le développement des macrophytes immergés déterminées par Sculthorpe (1967) et généralement admises. Il faut cependant rappeler que les limitations par la 
Tableau III. Quantités de matières en suspension (MEST, en mg/l) dans l'eau de l'étang du Vaccarès, mesurées lors de deux campagnes d'échantillonnage effectuées en 1965-66 et 1977.78.

\begin{tabular}{|c|c|c|c|c|}
\hline & Stations* & $\mathrm{n}$ & Moyenne/Ecart type & Etendue \\
\hline $\begin{array}{l}\text { Echantillonnage } \\
\text { (1965-1966) } \\
\text { Marazanof (1969) }\end{array}$ & $\begin{array}{r}7 \\
12 \\
18 \\
27 \\
14\end{array}$ & $\begin{array}{l}7 \\
7 \\
7 \\
7 \\
7\end{array}$ & $\begin{array}{r}75,0 / 105,2 \\
76,2 / 102,9 \\
122,2 / 133,4 \\
96,1 / 124,8 \\
85,4 / 93,0\end{array}$ & $\begin{array}{r}1,6 \cdot 276,0 \\
1,2-248,4 \\
23,6 \cdot 244,4 \\
2,0-327,6 \\
14,0-237,6\end{array}$ \\
\hline $\begin{array}{l}\text { Echantillonnage } \\
\text { (1977-1978) } \\
\text { Vaquer \& } \\
\text { Campredon } \\
\text { (inédit) }\end{array}$ & $\begin{array}{r}7 \\
12 \\
18 \\
11 \\
6\end{array}$ & $\begin{array}{l}11 \\
11 \\
11 \\
11 \\
11\end{array}$ & $\begin{array}{l}207,0 / 106,7 \\
256,5 / 133,7 \\
262,5 / 160,5 \\
244,4 / 130,0 \\
266,5 / 105,1\end{array}$ & $\begin{array}{c}88,0-460,0 \\
94,0-438,0 \\
75,0-709,0 \\
131,0-540,0 \\
138,0-498,0\end{array}$ \\
\hline
\end{tabular}

* Les numéros des stations correspondent à ceux at tribués lors de la campagne de 1984.

lumière peuvent être beaucoup plus variables suivant les espèces et les milieux comme l'ont observé Sheldon \& Boylen (1977). La valeur de $1 \%$ correspond au seuil de compensation au-delà duquel les dépenses énergétiques liées à la respiration dépassent l'énergie captée par la photosynthèse. L'examen du tableau 2 montre que les profondeurs moyennes calculées pour les deux niveaux 4 et $1 \%$ de lumière transmise, comprises entre $-2,04$ et $-2,89 \mathrm{~m}$, sont au moins égales ou supérieures à la profondeur maximale de l'étang. Il apparaît donc, au moins en 1984 et vraisemblablement pour tou te la période récente correspondant à la phase de resalement, que la lumière ne soit pas un facteur limitant pour la croissance des herbiers en dépit de mauvaises qualités optiques de l'eau.

A l'inverse, il n'en a vraisemblablement pas été de même pour les périodes précédentes en regard des quantités de matières en suspension totales déterminées sur le cycle annuel 1965-66 et de celles mesurées sur le cycle annuel 1977-78 (Tableau III). Cellesci atteignant respectivement 91 et $247,4 \mathrm{mg} . \mathrm{I}^{-1}$ en moyenne annuelle ont joué sans aucun doute un rôle déterminant pour le développement de la végétation immergée en lirnitant les quantités de lumière transmise dans la masse d'eau. En effet l'utilisation d'un modèle linéaire reliant les quantités de MEST aux pourcentages de lumière transmise à $0,1 \mathrm{~m}$ établi à partir de données recueillies en 1978 dans une rizière de Camargue (Pont \& Vaquer 1984) permet une approximation des quantités relatives de lumière transmises à différentes profondeurs dans l'étang pour les valeurs de MES déterminées à ces deux périodes. Ce modèle dont la forme est :

$$
\begin{aligned}
\mathrm{V} / \mathrm{Io}= & 98,6 . \mathrm{MEST}-246,8(\mathrm{n}=26 ; \mathbf{r}=0,92) \\
& (\% \text { à } 0,1 \mathrm{~m})
\end{aligned}
$$

dorme, pour les MES considérées, les valeurs de transmission à $0,1 \mathrm{~m}$ de $76,1 \%$ et $44,4 \%$. En entrant ces valeurs dans le modèle de Lambert-Beer, les profondeurs calculées du seuil de compensation sont respectivement de $1,7 \mathrm{~m}$ et $0,6 \mathrm{~m}$. Ces valeurs montrent clairement le rôle limitant de la lumière par l'intermédiaire des fortes teneurs en MEST pour le développement de la végétation aquatique, notamment pendant la période allant de 1969 à 1978 pendant laquelle les niveaux de salinité ont été les plus bas et sans aucune variation significative durant et entre les cycles annuels. Ces conditions étaient très favorables au maintien en suspension d'une grande quantité de particules.

\subsection{Les herbiers du Vaccarès}

Trois relevés de végétation effectués à 10 ans d'intervalle et cartographiés permettent de suivre l'évolution des herbiers dans l'étang depuis les années 60 jusqu'à la période actuelle.

La première carte établie à partir des relevés de Heurteaux \& Marazanof en 1963-64 (fig. 5) mont re la prédominance des herbiers à Potamogeton pectinatzs dans la partie nord et ouest de l'étang et de Ruppia maritima dans la partie sud. Quelques herbiers à Chara aspera (fa. marina) sont présents au niveau de Cabane Blanche, du mas d'Agon, à l'est 


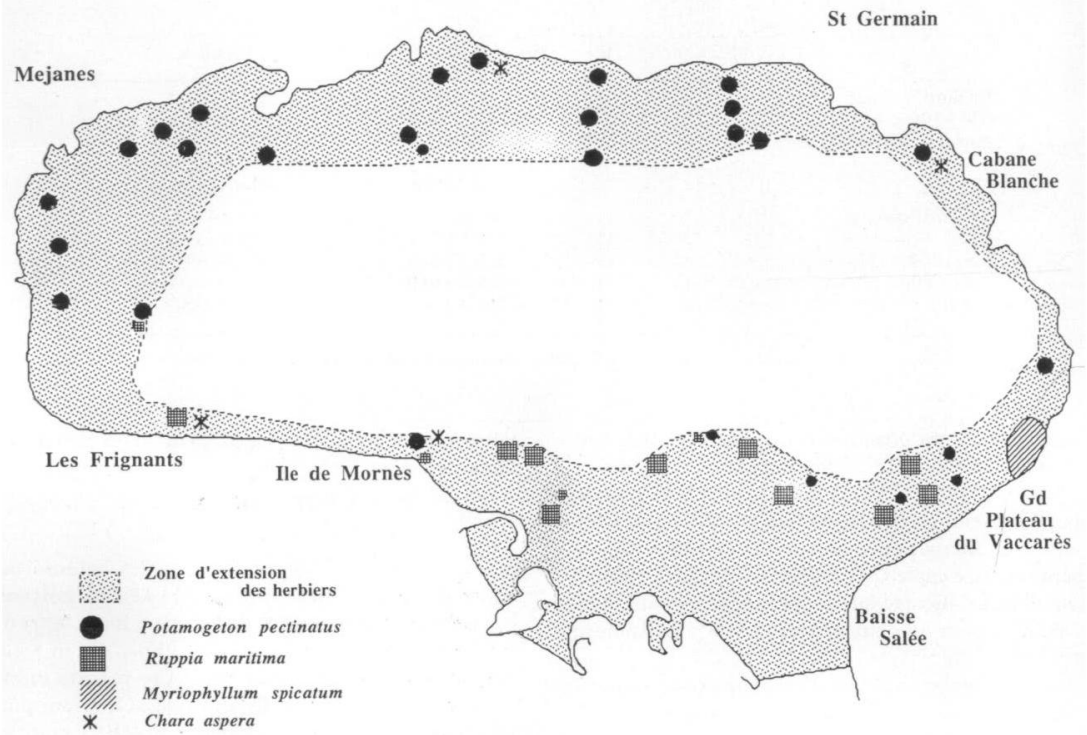

Fig. 5. Carte de répartition de la végétation et limite d'extension des herbiers établies à partir des observations de P. Heurteaux \& Marazanof en 1963 et 1964.

des Frignants et à la pointe de l'île de Mornès. Myriophyllum spicatum se développe dans une petite zone au N-E du Grand Plateau du Vaccarès. Cet te carte diffère de la carte phytosociologique de la Camargue de Molinier \& Devaux (1978) dessinée à partir de relevés effectués entre 1958 et 1966 , en ce que la limite des herbiers de Ruppia située par ces derniers sur un ligne Pointe de Mornès - Baisse salẻe s'étend bien au-delà suivant un axe t̂̉e de Mornès-Est du Grand plateau du Vaccarès. La zone d'extension des herbiers dans la zone littorale de l'étang est à peu près limitée par l'isobathe $-1,7 \mathrm{~m}$, ce qui correspond à la valeur du seuil de compensation calculée ci-dessus pour cette période.

La deuxième carte a été tracée à partir des rele. vés de végétation effectués par Vaquer \& Tamisier en août et septembre 1974 (fig. 6). Potamogeton pectinatus est toujours l'espèce dominante dans toutes les zones de l'étang, et particulièrement dans la partie Nord-Ouest et Ouest où elle constitue de vastes herbiers monospécifiques (Domaine de Méjanes, Carrelet, Mas Michel) ainsi que dans la partie sud le long de l'île de Mornès. Inversement les myriophy. les déjà présents en 63-64 se sont largement déve. loppés et forment avec les potamots des herbiers mixtes dans la partie Nord-Est à partir du mas d'Agon et la partie Est jusqu'à la baisse salée. Dans la zone sud menant vers les étangs du Fournelet et de Monro poussent quelques Ruppia cirrhosa. comme le signale également Verhoeven (1975). La zone des herbiers s'étend sur une bande côtière rela. tivement étroite, nettement plus réduite qu'en 63-64 


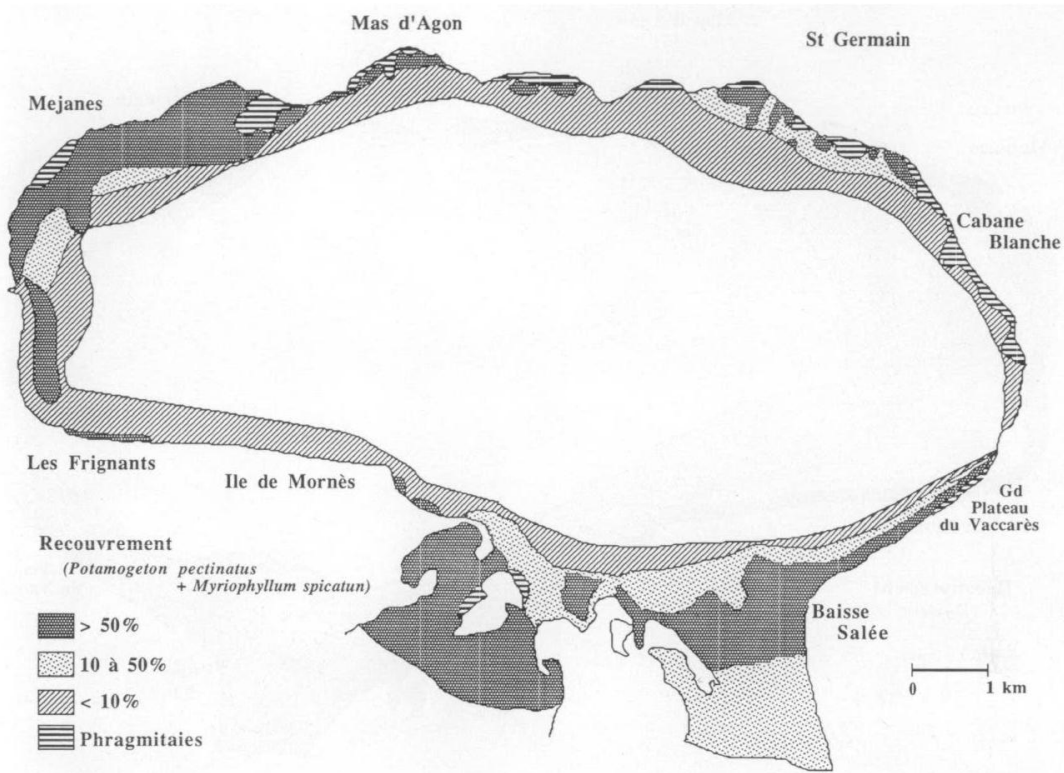

Fig. 6. Carte de répartition des herbiers d'après les relevés de végétation fails par A. Vaquer \& A. Tamisier en août et septembre 1974 .

sauf en quelques stations comme Notre Dame d'Amour, St Germain ou bien en bordu re de l'île de Momès. Bien que quelquess plantes aient pu être observées jusqu'à $1,8 \mathrm{~m}$ de profondeur, la grande majorité est située dans moins d'un mètre d'eau dans tou te la partie sud et à moins de $1,5 \mathrm{~m}$ partout ailleurs.

En 1984, les observations effectuées par Vaquer \& Bardou met tent en évidence le changement radical de la végétation qui est constituée en toutes zones uniquement par Zostera noltii (fig. 7). Cette espèce n'était apparemment pas ou peu connue en Camargue. Verhoeven \& Van Vierssen (1978) la signalent dans les étangs inférieurs de Camargue à partir de 1975. Cette végétation est accompagnée de Chaetomorpha linum qui forme par endroits de vastes accumulations plus ou moins flottantes à mi-eau.
L'invasion de l'étang du Vaccarès par ce macrophyte a débuté en 1981-82 après une phase de transition pendant laquelle tous les hydrophytes avaient été éliminés, à l'exception de quelques roselières sub. sistant à proximité des embouchures des émissaires d'eau de drainage tels que les canaux de Roquemaure et de Rousty. Une très large colonisation du pourtour de l'étang par des roselières à Phragmites commumis est à noter. Ces roselières qui avaient commencé à progresser dans les années 50 avec l'es. sor de la riziculture et parallèlement avec les apports croissants des eaux de drainage (Tallon 1954) ont même gagné l'étang de Monro, au sud, au niveau du radeau des Piles Palmes.

La surface occupée par les herbiers en 1984 plus vaste qu'en 1974 s'étend partout jusqu'à $1,8 \mathrm{~m}$ de profondeur sauf sur la rive nord entre le mas d'Agon 


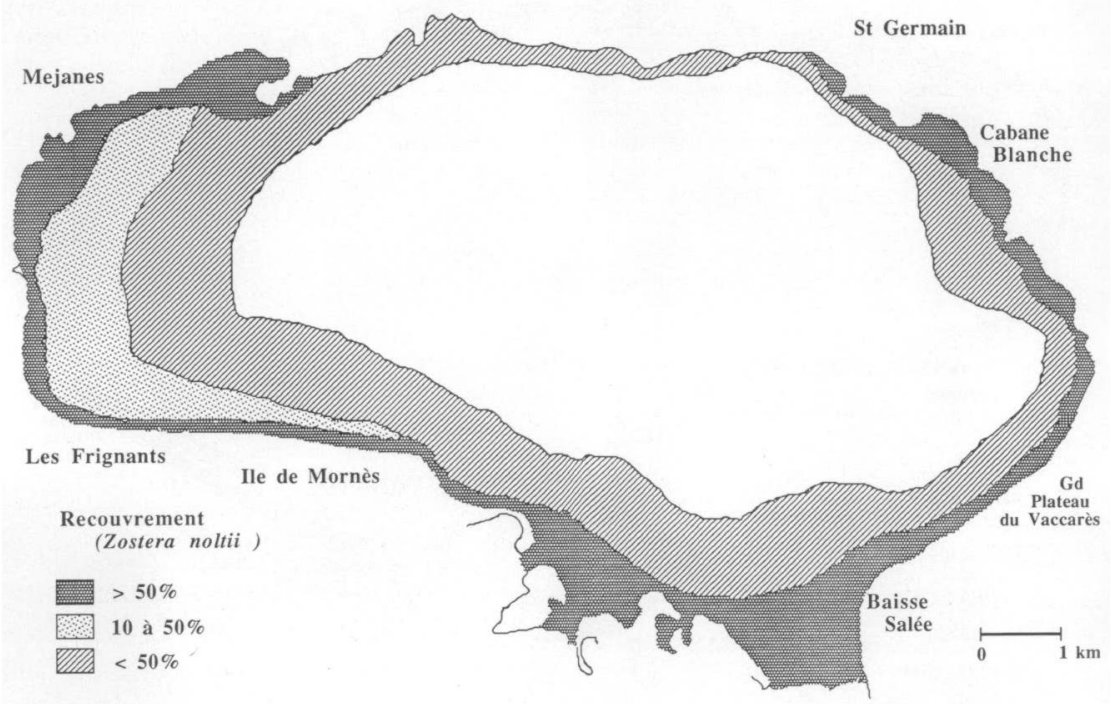

Fig. 7. Carte de la végétation de l'étang du Vaccarès levée par A. Vaquer \& G. Bardou en juillet et août 1984.

et St Germain où la végétation immergée ne dépasse pas 1,2 $\mathrm{m}$ de profondeur. En 1985, cet herbier s'est maintenu et quelques Ruppia cirrhosa sont obser. vées au nord de l'étang (Dervieux \& Tamisier 1987). La comparaison des cartes de 1974 et 1984 montre également, outre la plus grande extension des herbiers en 1984, que leur taux de recouvrement est plus élevé. En 1974, des recouvrements supérieurs à $50 \%$ sont notés seulement dans la partie est (Mejanes) et au sud, alors qu'un herbier dense est présent presque tout autour de l'étang en 1984.

\section{Discussion}

La salinité est le facteur prépondérant responsable des grandes transformations biologiques survenues au cours de ces dernières décennies. Depuis la mise en place de la digue à la mer au siècle dernier, ses variations ont été soumises progressivement à l'influence des activités humaines, et sa tendance vers un adoucissement s'est accrue avec l'emprise agricole comme le montrent les transformations observées avant 1950 (Tallon 1954, Petit \& Schachter 1954). Celles-ci ont affecté non seulement la végétation aquatique mais également la vie animale. Pendant la période de prospérité agricole, le dessalement s'est intensifié. Toutefois deux phases peuvent être distinguées. La première période s'étend de 1950 à 1970 pendant laquelle des espèces caractéristiques des biotopes saumâtres comme les Ruppia, Chara baltica (Tallon 1957) et Ch. aspera (Marazanof 1969) se maintiennent. Les deux dernières sont considérées respectivement comme des macroalgues des milieux saumâtres littoraux et alcalins à alcalino-saumâtres (Corillon 1975, Corillon \& 
Guerlesquin 1972). Puis de 1970 à 1978, la salinité diminue encore. Les Ruppia ont presque totalement disparu, les Phragmitaies atteignent leur maximum d'extension et les Myriophylles sont en plein essor. Le caractère saumâtre est encore marqué par la présence de certaines algues comme des Vaucheria (Vaquer 1976) et des Polyiphonia (Vaquer, inédit). L'absence de fluctuations saisonnières de salinité plus qu'une différence significative de salinité par rapport à la période précédente $\left(6,7 \mathrm{~g} . \mathrm{l}^{-t}\right)$ est sans doute à l'origine de l'évolution vers une végétation assez banale. Puis sous de nouvelles contraintes humaines, la tendance au dessalement s'est inversée depuis 1979, et les herbiers en place ont entièrement disparu et ont été remplacés par un herbier monospécifique de Zostera noltii qui s'est maintenu jusqu'à présent. Cette transformation est le deuxième bouleversement écologique majeur intervenu après celui lié à l'essor de la riziculture et consiste en un passage d'un bi otope légèrement saumâtre colonisé par des biocénoses dulçaquicoles à un biotope submarin habité par des organismes proches des milieux marins. Zostera noltii ainsi que $R$. cirrhosa occasionnellement observée en 1984 et 1985 poussent respectivement dans des gammes de salinité de 5 à 30 g.l-1 et 0,05 à 60 g.l-1 de $\mathrm{Cl}^{-}$(Verhoeven et al 1982).

De même que le développement des différentes espèces, la plus ou moins grande extension des herbiers peut être considérée, en partie, comme une conséquence indirecte de la salinité. La diminution de la surface colonisée $(50,3 \%$ en 1964 à $28,8 \%$ en 1974 de la surface de l'élang) pendant la phase d'adoucissement pourrait correspondre à une augmentation de la turbidité liée au maintien de la matière en suspension favorisée par l'eau douce. Pendant la première phase, les variations saisonnières de la salinité $\left(7,2 \mathrm{~g} \cdot \mathrm{I}^{-1}\right.$ en janvier pour $10,0 \mathrm{~g} . \mathrm{I}^{-1}$ en jujllet) auraient permis une diminution temporaire de la turbidité par floculation pendant l'été alors que pendant la période suivante l'absence de variations $\left(6,4\right.$ à 7,0 g. l-1 $\left.^{-1}\right)$ est sans doute à l'origine de la présence permanente d'une grande quantité de MEST. Ainsi pendant les phases d'adoucissement, la lumière peut être un facteur limitant pour le développement des herbiers. A l'inverse, avec l'augmentation de la salinité, cette limitation n'existe plus, et la surface colonisée augmente jusqu'à atteindre environ $44,7 \%$ en 1984 , valeur peu inférieure à celle observée en 1964. Si les conditions du milieu devaient se maintenir, il est vraisemblable que la surface occupée par l'herbier devrait encore augmenter d'autant que $Z$. noltii est une plante tout à fait bien adaptée aux il luminations extrêmes (Jimenez et al 1987) telles qu'elles peuvent régner dans ce milieu peu profond et actuellement bien éclairé. Cependant d'autres facteurs semblent pouvoir contenir cette extension puisqu'en l'absence de limitation par la lumière à certaines périodes (comme en 1964), la colonisation de l'étang reste incomplète. Parmi ceux-ci, la nature du substrat, dont les caractéristiques sont difficilement in terprétables en l'absence d'informations sur les courants animant la masse d'eau, notamment sous l'action des vents, pourrait jouer un rôle déterminant. C'est le cas notamment de la zone centrale où affleure un substrat compact, exempt de tout dépôt, où ne pousse aucune végétation.

L'évolution de la salinité de l'étang est difficile à prévoir. Elle dépend des possibles variations du stock de sels dissous dans l'ensemble du système (Vaccarès et étangs inférieurs). Actuellement (juillet 1988), celle-ci est de 17 g.l-1, soit la moitié de celle de janvier 1986. Cette salinité correspond à un stock de 2,3 millions de tonnes de sels totaux. Dans l'avenir, ce stock peut aussi bien continuer à décrốtre, augmenter ou rester stable. Il baissera si les fortes précipitations des hivers passés se renouvellent et si le nouvel essor rizicole persiste, ces deux facteurs sont favorables aux hauts niveaux et aux sorties d'eau salée par la digue à la mer. Au contraire, le stock de sel augmentera si une série d'années sèches provoque le maintien de basses eaux dans les étangs favorables aux apports souterrains de sels par la nappe aquifère. Le stock restera stable à l'échelle pluriannuelle en absence d'excès climatiques.

La végétation immergée est essentiellement tributaire de la salinité el de l'amplitude de ses varia tions. La tendance à la baisse de salinité déjà amorcée doit conduire à moyen terme à un nouveau changement de végétation, comme l'indique le développement de Ruppia cirrhosa observé au cours de l'été 1988. Ce changement conduira-t-il à une structure d'herbier connue ou non, à une plus grande diversité floristique? C'est un des problèmes intéressants posés par l'instabilité du milieu, caractéristique de l'étang du Vaccarès. Dans les mêmes termes, le problème peut sans doute se poser tant pour la microfaune invertébrée que pour la faune ichtyologique. 
Les réponses, s'il y en a, intéressent tout particulièrement les gestionnaires dé la Réserve Nationale de Camargue.

\section{T ravaux ciles}

Aguesse (P.) \& Marazanoff (F.). 1965. - Les modifications des milieux aquatiques de Camargue au cours des 30 dernieres années. Annls Limnol, 1(2): 163-190.

Dervieux (A.) \& Tamisier (A.). 1987. - Estimation par photointerprétation des surfaces couvertes par les herbiers immergés des plans d'eau de Camargue. Oecol. Applic., 8 (47): $371-385$.

Heurteaux (P.) \& Servant (J.). 1979. - Transferts et stockage de l'eau et des sels dans le profil pédologique des sols halomorphes camarguais. La Terre \& la Vie, Rev. Ecol. applic., (sppl.2) : $51-91$.

Jimenez (C.), Niell (F.X.) \& Algarra (P.). 1987. - Photosynthetic adaptation of Zostera noltii Homem. Aquatic Botany, 29 : 217.226.

Kirk (J.T.O.). 1980. - Relationship between nephelometry, turbidity and scattering coefficients in certain Australian waters. Aust. J, Mar. Freshwater Res., 31 : 1-12.

Marazanol (F.). 1969. - Contribution à l'étude écologique des mollusques des eaux douces et saumatres de Camargue. I. Milieux especces. Annls Limnol., 5(3): 201-323.

Molinier (R.) \& Devaux (J.P.). 1978. - Carte phytosociologique de la Camargue au 1/50000. Actes/Sud (ed.), F-13125 Le Paradou.

Pare Naturel Regional (ed.). 1986. - Bathymetrie du Vaccarès et des étangs inférieurs. Doc. polycop. cartés. Réserve Naturelle \& SNPN - GIS Camargue : 102 p
Petit (G.) \& Schachter (D.) 1954. - Notes sur l'évolution hydrolugique et écologique de l'étang du Vaccarès. La Terre et la Vie, $101 \cdot 121-128$

Pont (D.) \& Vaquer (A.). 1986. - Ouelques caractéristiques physico-chimiques de l'eau de submersion des rizières de Camargue (France) : róle de la végétation immergée et émergée. Ecologia Mediterrunea, 10 (1.2): 133-145

Sculthorpe (C.D.). 1967. - The biology of vascular plants. Edw. Amold (ed.), Londres : $610 \mathrm{p}$

Sheldon (R.B.) \& Boylen (C.W.). 1977. - Maximum depth inhabited by vascular plants. Am. Midl. Natur, $97: 248-254$.

Tallon (G.). 1954. - Transformation de la Camargue par la riziculture. Evolution du Vaccarès. La Terre \& la Vie, 101 : 65-79.

Tallon (G.). 1957. - Charophycèes de Camargue. La Terre \& Vie, $104: 120.121$

Vaquer (A.). 1976. - Le genre Vaucheria (Xanthophycées) dans l'ètang du Vaccarès et une sansouïre submergée voisine. $L a$ Terre \& la Vie, $4: 613-618$

Verhoeven (J.T.A.). 1975. - Ruppia-communities in the Camargue, France. Distribution and structure in relation to salinity and salinity fluctuations. Aquatic Botany; $1: 217.241$

Verhoeven (J.T.A.) \& Van Vierssen (W.). 1978. - Structure of macrophyte dominated communities in two brackish lagoons and the island of Corsica, France. Aquatic Botany, 5: 77-86.

Verhoeven (J.T.A.), Jacobs (R.P.W.M.) \& Van Vierssen (W.). 1982. - Life-strategies of aquatic plants : some critical notes and recommendations for further research. Studies on aquatic vascular plants. J. Symoens el al (ed.), Bruxelles, 158.164.

Waimsley (R.D.), Butty (M.), Dan Der Pieden (H.) \& Grobler (D.) 1980. - Light penetration and the interrelationship between optical parameters in a turbid subtropical impoundment. Hydrobiologia, 70 : 145-157. 